University of Nebraska - Lincoln

DigitalCommons@University of Nebraska - Lincoln

2011

\title{
Continuity of Landsat observations: Short term considerations
}

\author{
Michael A. Wulder \\ Pacific Forestry Centre, mwulder@nrcan.gc.ca \\ Joanne C. White \\ Pacific Forestry Centre \\ Jeffrey G. Masek \\ NASA Goddard Space Flight Center \\ John Dwyer \\ Center for Earth Resources Observation and Science (EROS) \\ David P. Roy \\ South Dakota State University, david.roy@sdstate.edu
}

Follow this and additional works at: https://digitalcommons.unl.edu/nasapub

Part of the Physical Sciences and Mathematics Commons

Wulder, Michael A.; White, Joanne C.; Masek, Jeffrey G.; Dwyer, John; and Roy, David P., "Continuity of Landsat observations: Short term considerations" (2011). NASA Publications. 98.

https://digitalcommons.unl.edu/nasapub/98

This Article is brought to you for free and open access by the National Aeronautics and Space Administration at DigitalCommons@University of Nebraska - Lincoln. It has been accepted for inclusion in NASA Publications by an authorized administrator of DigitalCommons@University of Nebraska - Lincoln. 


\title{
Continuity of Landsat observations: Short term considerations
}

\author{
Michael A. Wulder ${ }^{\text {a,*}}{ }^{\text {, Joanne C. White }}{ }^{\text {a }}$, Jeffrey G. Masek ${ }^{\text {b }}$, John Dwyer ${ }^{c}$, David P. Roy ${ }^{\text {d }}$ \\ a Canadian Forest Service, Pacific Forestry Centre, Natural Resources Canada, Victoria, BC, V8Z 1M5, Canada \\ b Biospheric Sciences Branch, NASA Goddard Space Flight Center, Greenbelt, MD, USA \\ c United States Geological Survey, Center for Earth Resources Observation and Science (EROS), 47914 252nd Street, Sioux Falls, SD 57198-0001, USA \\ ${ }^{d}$ Geographic Information Science Center of Excellence, South Dakota State University, Brookings, SD 57007, USA
}

\section{A R T I C L E I N F O}

\section{Article history:}

Received 27 August 2010

Received in revised form 1 November 2010

Accepted 6 November 2010

\section{Keywords:}

Landsat

Monitoring

Operational

Research

Continuity

Global

Land cover

Change detection

\begin{abstract}
A B S T R A C T
As of writing in mid-2010, both Landsat- 5 and -7 continue to function, with sufficient fuel to enable data collection until the launch of the Landsat Data Continuity Mission (LDCM) scheduled for December of 2012. Failure of one or both of Landsat- 5 or -7 may result in a lack of Landsat data for a period of time until the 2012 launch. Although the potential risk of a component failure increases the longer the sensor's design life is exceeded, the possible gap in Landsat data acquisition is reduced with each passing day and the risk of Landsat imagery being unavailable diminishes for all except a handful of applications that are particularly data demanding. Advances in Landsat data compositing and fusion are providing opportunities to address issues associated with Landsat-7 SLC-off imagery and to mitigate a potential acquisition gap through the integration of imagery from different sensors. The latter will likely also provide short-term, regional solutions to application-specific needs for the continuity of Landsat-like observations. Our goal in this communication is not to minimize the community's concerns regarding a gap in Landsat observations, but rather to clarify how the current situation has evolved and provide an up-to-date understanding of the circumstances, implications, and mitigation options related to a potential gap in the Landsat data record.
\end{abstract}

Crown Copyright @ 2010 Published by Elsevier Inc. All rights reserved.

\section{Introduction}

Landsat imagery of some form has been collected since 1972, resulting in the longest continuously acquired collection of spacebased terrestrial observation. The spatial resolution of the imagery is informative of human activities on the Earth's surface (Townshend \& Justice, 1988) and has made Landsat imagery an invaluable information source for science, management, and policy development (Goward \& Masek, 2001). Further, the opening of the entire U.S. Geological Survey (USGS) Landsat archive in 2008 and 2009 (USGS, 2008), which made all of the USGS Landsat imagery freely available through a web-portal (glovis.usgs.gov) (Woodcock et al., 2008), has resulted in an increased capacity to undertake ambitious analyses of terrestrial dynamics across large areas, and using dense time series of imagery. Cohen and Goward (2004) provide an historical overview of the Landsat program and Landsat data applications.

Although both Landsat- 5 and -7 continue to function and have sufficient fuel to enable data collection until the Landsat Data Continuity Mission (LDCM) is launched in December 2012 and begins operations, both missions have met with serious challenges in recent years (Beck, 2005; Markham et al., 2004; USGS, 2009). As a result, there is the potential for a gap in the extensive Landsat observation

\footnotetext{
* Corresponding author.

E-mail address: mwulder@nrcan.gc.ca (M.A. Wulder).
}

record, should one or both of Landsat-5 or -7 fail before LDCM is launched and considered operational. The goal of this communication is provide an up-to-date understanding of the situation, note the implications of a Landsat data gap for operational users, and present possible mitigation options.

\section{Landsat sensor and acquisition situation}

\subsection{Landsat-5 and -7}

Landsat-5 was launched on March 1, 1984 and had a five-year design life. Landsat-6 was launched on October 5, 1993 but did not achieve orbit. Landsat-7 was launched on April 15, 1999, and had a design life of 5 years. Both Landsat- 5 and -7 continue to function and acquire data. Landsat- 5 is beset with issues one would expect of an aging satellite lasting well beyond its design life, with numerous interventions of a creative engineering team largely focused on managing dated spacecraft electronics needed for the sensor's data to be relayed to ground stations (Beck, 2005; USGS, 2009). Two issues will limit the longevity of Landsat-5's remaining mission lifetime. First, the operating current within the Traveling Wave Tube Amplifier (part of the data transmission segment) has been rising steadily since early 2010. If this rise continues, an over-current condition (and termination of data transmission) could occur before the launch of LDCM. Second, sufficient fuel exists for just one more set of inclination maneuvers (which are currently scheduled for late October 2010, 
putting this consideration in the past by time of publication); after which, the equatorial crossing time for the satellite will begin to drift and by 2014 , the crossing time will be too early in the day for useful land observations. It is worth noting that Landsat-5, based upon the capacity and operation of ground receiving stations, no longer has global coverage.

Landsat-7 continues to operate, albeit, since May 31, 2003, with a failed scan line corrector (SLC). The SLC compensates for the forward motion of the sensor and its failure has resulted in images that have high geometric and radiometric fidelity, but no data present for wedges varying in size from one $30 \mathrm{~m}$ pixel near the centre of the image to fourteen $30 \mathrm{~m}$ pixels along the eastern and western edges of the image (Storey et al., 2005). Although the central swath of the Landsat-7 image (approximately $22 \mathrm{~km}$ wide) is not impacted by the SLC failure, approximately $22 \%$ of the image data are lost. To mitigate the impact of the data gaps caused by the SLC failure, a number of approaches have been developed, including image segmentation (Maxwell et al., 2007) and multi-date (same season) image compositing. Subsequent investigations have determined that the segment-based gap-filled SLC-off imagery is sufficiently robust for certain land cover applications (Bédard et al., 2008; Wulder et al., 2008a), while the multi-date compositing has been used for the NASA/USGS Global Land Survey (GLS) product suite (Gutman et al., 2008). Image compositing has also provided an opportunity to both address the data gaps related to SLC-off and to enable cloud infill for seamless, wide-area, characterizations (Lindquist et al., 2008; Roy et al., 2010). Landsat-7 has sufficient fuel to maintain operations through 2016.

\subsection{LDCM status and plans}

The LDCM will have the same orbit as previous Landsat missions and will carry two sensors: the Operational Land Imager (OLI) and the Thermal Infrared Sensor (TIRS). The OLI will have similar spatial, spectral, and temporal characteristics to the Thematic Mapper (TM) and Enhanced Thematic Mapper Plus (ETM+) sensors; however, the OLI will be a pushbroom instrument-a key difference from previous Landsat sensors. The pushbroom concept for OLI was prototyped by the Advanced Land Imager (ALI) on the NASA platform EO-1 (Ungar et al., 2003) and will provide improved geometric fidelity, and enable a longer detector dwell time, thereby improving signal-to-noise. Unlike the previous Landsat missions, which collected imagery using 8-bit quantization, the OLI will collect imagery utilizing 12-bit quantization. This improved radiometric resolution matches that of the MODIS (Xiong et al., 2005) and will provide greater dynamic range than previous Landsat sensors and reduce saturation problems associated with globally maximizing the range of land surface spectral radiance into a limited 8-bit range (Markham et al., 2006).

The spectral bandwidths of the OLI will be slightly different from those of TM and ETM+ in order to minimize specific atmospheric absorption features; the OLI will have two additional bands, a coastal aerosol band centered at $443 \mathrm{~nm}$, designed to aid coastal water quality investigations, and a cirrus band centered at $1375 \mathrm{~nm}$, designed to detect cirrus cloud contamination. The 8 multispectral bands of the OLI will have a spatial resolution of $30 \mathrm{~m}$, and the panchromatic band will have a spatial resolution of $15 \mathrm{~m}$. The TIRS will collect data in two narrow thermal bands (centered at 10,800 and 12,000 nm) positioned in the same thermal region that was previously covered by the thermal high and low gain bands on Landsats 4-7 (Jhabvala et al., 2009). TIRS data will have a 12-bit quantization and a $100 \mathrm{~m}$ spatial resolution. The data from all 11 spectral bands will be co-registered to create an LDCM data product containing both OLI and TIRS data.

Advances in onboard recording and data downlink capacity will enable all the LDCM data (approximately 400 scenes per day) to be gathered centrally by a receiving station at the USGS Earth Resources Observation and Science Center (EROS). This will provide centralization of imagery and improved global coverage compared to ETM+ data, which was specified to collect 250 to 300 scenes per day (Arvidson et al., 2001) with more acquired after the SLC failure, due to the lack of international station receiving utilization, towards the instrument limit of 300 to 350 per day. With the increased LDCM acquisition rate, the probability of acquiring cloud-free images in a given time period will be greater than ETM+ (Ju \& Roy, 2008). The onboard transmitting systems also provide the capacity to broadcast real time from the LDCM satellite via an X-band link to a network of international receiving stations as required. Additional information on the LDCM and the OLI and TIRS sensors can be found at: http://ldcm.usgs.gov/.

At present, the LDCM design has passed a critical design review and is on-track for the proposed launch date of December 2012 launch (DeWitt \& Beck, 2010). A number of months have been built into the current launch schedule by the NASA mission planning team to aid in addressing unforeseen technical issues in sensor development, integration, and testing.

\section{Gap scenarios and mitigation opportunities}

At the time of writing, both Landsat-5 and -7 continue to operate, acquisition for the 2010 growing season in the northern hemisphere is complete, and the growing season in the southern hemisphere has yet to start. Immediate and catastrophic failure of both Landsat-5 and -7 instruments would lead to a possible gap of two northern hemisphere growing seasons (i.e. 2011 and 2012) and three southern hemisphere growing seasons (i.e. 2010, 2011, and 2012) before the availability of LDCM data in early 2013. For some applications, the possibility of having no data for 2 or 3 years may not cause significant hardship (e.g., archaeological investigations), while for others, this lack of data would be extremely problematic (e.g., monitoring of tropical deforestation). In a broader context, the impacts of either Landsat-5 or -7 failing are not similar, as Landsat-7 provides global coverage (with SLC-off), while Landsat-5 does not. Currently, the collection of global data by Landsat-5 is no longer possible due to an insufficient number and distribution of international ground receiving stations.

The opportunities for mitigating the impact of this potential gap in Landsat data availability are framed by the specific information needs of a given application. Some applications require imagery from every 16-day overpass of the Landsat satellite (i.e., water resource management, phenological studies), while others require a single annual image to aid with land cover mapping and/or change monitoring (Wulder et al., 2008b). Applications with more intensive data needs are less likely to be able to mitigate the impact of a data gap; however for other applications, there may be several options, including alternate data from different medium resolution sensors, compositing, or data fusion and generation of synthetic imagery. The sections below detail these mitigation alternatives.

\subsection{Alternate imagery}

Landsat is unique in offering a high spatial resolution (30 m) over a large image footprint $(185 \times 185 \mathrm{~km})$ with high quality and calibrated radiometric characteristics (Wulder et al., 2008b). Landsat data are also collected following a long-term acquisition plan (LTAP) to ensure global coverage (Arvidson et al., 2001) with collected imagery stored and made available through an open access archive (Woodcock et al., 2008). To mitigate a possible gap in Landsat imaging, NASA and the USGS convened an interagency Data Gap Study Team, which identified alternate data sources (focusing on IRS, CBERS) and also characterized the spectral, radiometric, geometric, and spatial characteristics of these sources (Chander, 2007). While Chander (2007) summarize these characteristics in detail, that include baseline specifications for spectral bands, $<15 \%$ error in at-sensor radiance, pixels sized 10 to $100 \mathrm{~m}$ pixel dimensions, geographic and band-to-band registration targets, and global observation of all land areas between $\pm 81.2^{\circ}$ latitude at least twice per year. Powell et al. (2007) also identify the 
criteria required for a particular sensor to be considered similar to Landsat. Several programs and sensors are identified (e.g., SPOT, IRS, CBERS, ASTER, and ALI) as having the potential to address a gap in Landsat operations. While these communications can be consulted for lists of sensor characteristics and performance, from an applications point of view, specific user needs should guide the selection of alternate data source (Wulder et al., 2008b). For instance, even if spatial, spectral, and temporal characteristics are appropriate, users should be mindful of the implications of smaller image footprints (leading to increased data management requirements and variable view angles between adjacent images), downlink capabilities, image availability (on-demand or systematic collection and archiving), access to the imagery, and capacity to share unencumbered by restrictive data policies.

To note ongoing developments, the Committee on Earth Observation Satellites (CEOS) is working to develop, through a Virtual Constellation concept, the capacity to incorporate the assets of various space programs to produce coordinated and complementary observations. Of pertinence to the continuity of observations in support of terrestrial ecosystem characterization, the CEOS Virtual Constellation for Land Surface Imaging is aligning applicable space agencies to maximize the integration of current satellite-based observations and to recommend appropriate future missions (Loveland et al., 2008).

Currently there are no other missions analogous to Landsat that have global observation capabilities or accumulated global archives. In cases where there are data that have been or could be acquired to augment Landsat holdings, data sharing agreements and political considerations can hinder such activities. Sensors from non-Landsat missions may meet some baseline requirements to emulate Landsat image characteristics, but it is unlikely that sufficient similarity exists to enable direct integration or interoperability, especially from operations perspectives where known relationships and algorithms will no longer function.

\subsection{Compositing}

Compositing has been used as a practical way to reduce residual aerosol and cloud contamination, fill missing pixel values, and reduce the data volume of moderate resolution near-daily coverage sensor data such as AVHRR or MODIS (Cihlar, 1994; Holben, 1986; Roy, 2000). Compositing approaches based on BRDF model inversion, such as the MODIS Nadir BRDF-Adjusted Reflectance (NBAR) product (Ju et al., 2010; Schaaf et al., 2002) are not appropriate for Landsat application because the 16-day repeat cycle and narrow $15^{\circ}$ field of view of the Landsat sensors do not provide a sufficient number, or angular sampling of the surface to reliably invert BRDF models against Landsat reflectance (Danaher et al., 2001; Roy et al., 2010), and they do not provide a solution for compositing the thermal bands. Consequently, compositing based on the selection of a "best" pixel over the compositing period (time series of image acquisitions) is more appropriate for Landsat application. We note that there are problematic situations for compositing if there is a paucity of imagery. In the same way that imagery for a given place or time may not be available and not capture the desired seasonal conditions, these situations will also result in a lack of that condition or property for compositing purposes (Ju et al., 2010). Use of imagery from differing times of the year in composite development may result in spatially incoherent composites that require normalization. In locations where rapid change is on-going, compositing could inadvertently incorporate these changed conditions into the composite (Roy et al., 2008).

Compositing of multi-temporal Landsat thematic data classifications has been demonstrated as a means to generate wide-area mosaics of classification data (Guindon \& Edmonds, 2002). Lindquist et al. (2008) and Roy et al. (2010) developed the concept of Landsat compositing to create monthly, seasonal, and annual continental composited mosaics, by selecting from multiple ETM+ observations of the same pixel the observation that is not cloud contaminated. If several observations meet this criterion, the observation with the maximum NDVI is selected. For certain areas that have low vegetation cover, the observation with the maximum brightness value is selected. This compositing approach could be extended to include imagery from different sensors, thereby further increasing the options to mitigate a Landsat data gap. Efforts to composite data acquired by different Landsat sensors will be supported by cross-calibration activities producing radiometric calibration coefficients for Landsat's MSS, TM, and ETM+ (Chander et al., 2009).

\subsection{Data fusion and synthetic image generation}

Fusion of remotely sensed data acquired from different satellite systems allows for exploitation of the sensors' different spectral, spatial, angular, and temporal sensing characteristics (Pohl \& Van Genderen, 1998). Fusion can be used to fill SLC-off Landsat ETM+ data gaps and gaps caused by clouds, radiometrically normalize Landsat data, and provide Landsat-like synthetic data. Data fusion can be undertaken in a purely empirical manner, for example, by extraction and compositing of spectral vegetation indices derived from different systems (van Leeuwen et al., 2006), or by weighting reflectances from different sensors according to their spectral differences (Gao et al., 2006). Alternatively, data fusion can be undertaken using more physically-based approaches, for example by inversion of BRDF models (Roy et al., 2008). Reliable data fusion requires that the data from each system are precisely co-registered, calibrated, spectrally normalized to common wavebands, and atmospherically corrected using appropriate atmospheric characterization information, although the requirement for common wavebands can potentially be relaxed if physically-based reflectance models are used for data fusion (Pinty et al., 2004).

The radiometric consistency of Landsat data may change due to sensor calibration changes, atmospheric, cloud and shadow contamination, and differences in illumination and observation angles (Danaher et al., 2001; Roy et al., 2010; Song et al., 2001; Toivonen et al., 2006). This variation can be modeled by fitting a reflectance function parameterized for cross-track location (Hansen et al., 2008) or spectral regression functions (Gao et al., 2010; Olthof et al., 2005) derived by examination of contemporaneous satellite data from a different source. Empirical functions of this sort can be used to predict reflectance. For example, Gao et al. $(2006,2010)$ generate synthetic Landsat like imagery by combining a base Landsat image from one date with MODIS imagery from the date in question. The result of the data blending is a synthetic-Landsat image that maintains Landsat spatial resolution with the temporal characteristics guided by the MODIS imagery. Hilker et al. (2009a) has shown in an applications context that the spectral change expected over a growing season is captured with the synthetic imagery. Hilker et al. (2009b) extended the initial data blending logic of Gao et al. (2006) to produce dated change over a time series of synthetic data: a Landsat image pair is used to spatially guide a MODIS change algorithm to enable the attribution of change date. The change detection and dating protocol of Hilker et al. (2009b) only requires a high spatial resolution change mask, so imagery from different sensors can be used to identify change (Wulder et al., 2008c).

Roy et al. (2008) used a semi-physical fusion approach and the MODIS BRDF/albedo land surface characterization product to predict Landsat ETM+ reflective wavelength reflectance on the same, an antecedent, or subsequent date and demonstrated that this approach may be used for cloud and SLC-off gap filling and for radiometric normalization. This BRDF approach may be applied to any high spatial resolution satellite data and does not require empirical tuning parameters or regression function derivation and therefore may be automated. Furthermore, this approach accommodates temporal variations due to surface changes (e.g., phenological, land cover/land use variations) observable at the $500 \mathrm{~m}$ MODIS BRDF/Albedo product 
resolution. If either Landsat-5 or -7 should fail, synthetic image data from a Landsat base image date could be developed for a period of time using these aforementioned approaches. Users should be mindful that synthetic images are not a replacement for actual Landsat images, with lessened geometric and radiometric qualities, but are an option for producing imagery to meet operational requirements. Aside from continuity of medium spatial resolution observations, applications requiring both dense spatial and temporal characterization may be aided by these image processing opportunities.

\section{Discussion}

At the present time, it is entirely possible that both Landsat-5 and Landsat-7 will still be operational at the time of the LDCM launch; however, a failure of either satellite is possible prior to the launch of LDCM in December 2012. If Landsat-5 fails (the most likely scenario given the satellite's age), Landsat-7 SLC-off segmentation (e.g., Maxwell et al., 2007) or compositing (Roy et al., 2010) can continue to provide useful Landsat gap filled image data.. A Landsat-7 failure will leave Landsat-5 operational; however, as Landsat-5 was launched in 1984 with a projected 5 year design life, data users should not be surprised by an eventual failure of Landsat- 5 and should plan accordingly. For instance, mindful of a potential gap in continuity of Landsat imagery and for other agricultural monitoring specific benefits (i.e., wider swath, more frequent acquisition), some US agencies, such as the Department of Agriculture (USDA), have been mitigating risk by procuring alternate imagery. For example, the USDA has been integrating AWIFS into monitoring operations since 2006 following a research and transition period to accommodate this alternate image source. Johnson (2008) provides a classification comparison between Landsat-5 TM and AWIFS resulting in an informative summary of the applications utility of AWIFS data from an agricultural monitoring perspective, highlighting the importance of increased temporal frequency and a wide field of view. Increased temporal frequency aids in collecting cloud free observations and enables alternate spatial and temporal data processing options. The wide field of view, $737 \mathrm{~km}$ swath versus $185 \mathrm{~km}$ for Landsat, enables efficient and consistent large area characterizations, albeit with a loss in spatial detail due to a $56 \mathrm{~m}$ spatial resolution.

The impact of a failure of Landsat- 5 or -7 would be further exacerbated by any slippage in the launch of the LDCM. Additionally, the lack of a back-up system or sensor poised for launch if there are problems with LDCM could lead to a significant data gap, highlighting the need to undertake steps to construct the successor to LDCM.

Alternate data sources that provide data analogous to Landsat may be utilized depending on the application and the information need. The dense time series of sensors such as MODIS may also be used to monitor change, conceding insensitivity to detection of smaller or isolated change events (Wulder et al., 2010). Temporal compositing provides opportunities to enhance the use of Landsat-7 imagery. Fusion provides unique opportunities to create synthetic-Landsat data based upon lower spatial resolution yet more frequently collected data. Some users may be able to use coarser spatial resolution data to monitor change, perhaps even to date larger change events (following Hilker et al., 2009b) and then subsequently follow-up with a retrospective audit of change once LDCM has been launched.

In the future, Landsat systems should be launched at shorter intervals to ensure data continuity. Consideration should be given to having multiple Landsat systems in orbit at a given time, or to having a system built and ready for launch should such need arise. The ideal solution would be to launch new missions at 5-year intervals with a 10-15 year design life, thus increasing the frequency of repeat coverage and minimizing data gaps due to component failures. Further, options to integrate observations from lower-cost sensors with the Landsat data could be explored, with Landsat serving as a reference standard (for geometry, radiometry, etcetera) and the lower cost systems providing denser coverage as well as a continuity of observations.

Additional "reference" missions would also bolster data continuity. The European Space Agency is planning to launch a pair of Sentinel-2 missions that deploy a sensor with imaging characteristics similar to that of LDCM, with the first mission scheduled for launch in 2013. With a larger image extent than Landsat (with a $290 \mathrm{~km}$ swath) and plans for two satellites to be launched for concurrent operation the capacity for landscape-scale terrestrial characterizations globally is enhanced. The potential for NASA and the USGS to work with the ESA to harmonize across programs to ensure long-term overlap in observations (continuity) and to aid in enabling global coverage is also present. Development of a long term acquisition plan (LTAP) that incorporates observations across sensors would aid in ensuring global and seasonal coverage while also enabling an increase in acquisitions over persistently cloudy regions. The ESA has announced intentions of an open data policy analogous to that of Landsat, although details have yet to be determined (deSelding, 2010).

Through this communication we do not wish to understate the tenuous state of the current Landsat missions; our intent is to indicate the current mission status and to be open of the mission status and to communicate possible opportunities. Further, the on-going intention for singular Landsat missions does not sufficiently mitigate the risk to acquisitions that have borne out over the life of the Landsat missions. As evidenced by Landsat-6, failure at launch can occur. Multiple Landsat class satellites will increase the effective temporal resolution of observations, and as the satellites have different overpass time will increase the opportunity for cloud free observations, and so increased data for compositing, and a reduction of risk to data gap through a critical Landsat failure. A goal of multiple concurrently operating Landsat satellites, or complementary satellites that may be lower cost but that buttress against the high standards of Landsat geometric and radiometric characteristics should be seriously considered.

We recommend that Landsat users look at their particular research and programmatic needs for Landsat imagery with an understanding that the loss of observational continuity is possible before 2012, but also that the probability for this data gap is diminishing. User needs for data and information need to be communicated to the USGS Land Remote Sensing Program. It is also recommend that policy and institutional impediments to the continuity of Landsat observations are identified and addressed.

\section{Acknowledgements}

We gratefully acknowledge USGS and NASA leadership and support of the Landsat Science Team.

\section{References}

Arvidson, T., Gasch, J., \& Goward, S. N. (2001). Landsat-7's long-term acquisition planAn innovative approach to building a global imagery archive. Remote Sensing of Environment, 78(1-2), 13-26.

Beck, R. (2005). Landsat-5 experiencing technical difficulties. USGS Press Release, (November 30, 2005). Available from. http://landsat.gsfc.nasa.gov/pdf_archive/ 15_solar_drive_press_release.pdf [accessed (July 26, 2010)].

Bédard, F., Reichert, F., Dobbins, R., \& Trépanier, I. (2008). Evaluation of segment-based gap-filled Landsat ETM + SLC-off satellite data for land cover classification in southern Saskatchewan, Canada. International Journal of Remote Sensing, 29(7) $2041-2054$.

Chander, G. (2007). Initial data characterization, science utility, and mission capability evaluation of candidate Landsat mission data gap sensors. Landsat Data Gap Study Version 1.0. Available from. http://calval.cr.usgs.gov/documents/LDGST_Technical_Report6.pdf [accessed August 25, 2010)].

Chander, G., Markham, B. L., \& Helder, D. L. (2009). Summary of current radiometric calibration coefficients for Landsat MSS, TM, ETM+, and EO-1 ALI sensors. Remote Sensing of Environment, 113, 893-903.

Cihlar, J. (1994). Detection and removal of cloud contamination from AVHRR images. IEEE Transactions on Geoscience and Remote Sensing, 32(3), 583-589.

Cohen, W. B., \& Goward, S. N. (2004). Landsat's role in ecological applications of remote sensing. Bioscience, 54(6), 535-545. 
Danaher, T., Wu, X., \& Campbell, N. (2001). Bi-directional reflectance distribution function approaches to radiometric calibration of Landsat TM imagery. Proceedings of the IEEE Geoscience and Remote Sensing Symposium (IGARSS 2001), July 9-13 2001 Sydney, Australia, Vol. 6. (pp. 2654-2657).

deSelding, P. B. (2010). European officials embrace open data policy for GMES Satellites Space News (June 30, 2010). Available from. http://www.spacenews.com/ civil/100630-officials-open-data-policy-gmes.html [accessed (August 25, 2010)].

DeWitt, S., \& Beck, R. (2010). NASA completes critical design review of Landsat Data Continuity Mission. Goddard Press Release No. 10-052 (June 1, 2010). Available from. http://landsat.gsfc.nasa.gov/news/news-archive/news_0285.html [accessed (August 25, 2010)].

Gao, F., Masek, J., Schwaller, M., \& Hall, F. (2006). On the blending of the Landsat and MODIS surface reflectance: predicting daily Landsat surface reflectance. IEEE Transactions on Geoscience and Remote Sensing, 44(8), 2207-2218.

Gao, F., Masek, J., Wolfe, R., \& Huang, C. (2010). Building consistent medium resolution satellite data set using MODIS products as reference. Journal of Applied Remote Sensing, 4, 043526, doi:10.1117/1.3430002.

Goward, S. N., \& Masek, J. G. (2001). Editorial: Landsat-30 years and counting. Remote Sensing of Environment, 78(1-2), 1-2.

Guindon, B., \& Edmonds, C. M. 2002. Large-area land-cover mapping through scenebased classification compositing. Photogrammetric Engineering \& Remote Sensing. Vol. 68, No. 6, pp. 589-596.

Gutman, G., Byrnes, R., Masek, J., Covington, S., Justice, C., Franks, S., et al. (2008). Towards monitoring land-cover and land-use changes at a global scale: The Global Land Survey 2005. Photogrammetric Engineering and Remote Sensing, 74(1), 6-10.

Hansen, M. C., Roy, D. P., Lindquist, E., Adusei, B., Justice, C. O., \& Altstatt, A. (2008). A method for integrating MODIS and Landsat data for systematic monitoring of forest cover and change and preliminary results for Central Africa. Remote Sensing of Environment, 112(5), 2495-2513.

Hilker, T., Wulder, M. A., Coops, N. C., Seitz, N., White, J. C., Gao, F., et al. (2009a). Generation of a dense time series synthetic Landsat data through data blending with MODIS using a spatial and temporal adaptive reflective fusion model. Remote Sensing of Environment, 113(9), 1988-1999.

Hilker, T., Wulder, M. A., Coops, N. C., Linke, J., McDermid, G., Masek, J., et al. (2009b). A new data fusion model for high spatial- and temporal- resolution mapping of forest disturbance based on Landsat and MODIS. Remote Sensing of Environment, 113(8), $1613-1627$.

Holben, B. (1986). Characteristics of maximum-value composite images from temporal AVHRR data. International Journal of Remote Sensing, 7(11), 1417-1434.

Jhabvala, M., Reuter, D., Choi, K., Jhabvala, C., \& Sundaram, M. (2009). QWIP-based thermal infrared sensor for the Landsat Data Continuity Mission. Infrared Physics $\mathcal{E}$ Technology, 52(6), 424-429.

Johnson, D. M. (2008). A comparison of coincident Landsat-5 TM and Resourcesat-1 AWiFS imagery for classifying croplands. Photogrammetric Engineering and Remote Sensing, 74(11), 1413-1423.

Ju, J., \& Roy, D. P. (2008). The availability of cloud-free Landsat ETM+ data over the conterminous United States and globally. Remote Sensing of Environment, 112(3), 1196-1211.

Ju, J., Roy, D. P., Shuai, Y., \& Schaaf, C. (2010). Development of an approach for generation of temporally complete daily nadir MODIS reflectance time series. Remote Sensing of Environment, 114(1), 1-20.

Lindquist, E., Hansen, H., Roy, D. P., \& Justice, C. O. (2008). The suitability of decadal image data sets for mapping tropical forest cover change in the Democratic Republic of Congo: Implications for the mid-decadal global land survey. International Journal of Remote Sensing, 29(24), 7269-7275.

Loveland, T. R., Cochrane, M. A., \& Henebry, G. M. (2008). Landsat still contributing to environmental research. Trends in Ecology E' Evolution, 23(4), 182-183.

Markham, B. L., Storey, J. C., Williams, D. L., \& Irons, J. R. (2004). Landsat sensor performance: History and current status. IEEE Transactions on Geoscience and Remote Sensing, 42(12), 2691-2694

Markham, D., Goward, S., Arvidson, T., Barsi, J., \& Scaramuzza, P. (2006). Landsat-7 longterm acquisition plan radiometry-evolution over time. Photogrammetric Engineering and Remote Sensing, 72(10), 1129-1135.

Maxwell, S. K., Schmidt, G. L., \& Storey, J. C. (2007). A multi-scale approach to filling gaps in Landsat ETM+ SLC-off images. International Journal of Remote Sensing, 28(23), 5339-5356.

Olthof, I., Butson, C., Fernandes, R., Fraser, R., Latifovic, R., \& Orazietti, J. (2005). Landsat ETM+ Mosaic of Northern Canada. Canadian Journal of Remote Sensing, 31(5) $412-419$.
Pinty, B., Taberner, M., Liang, S., Govaerts, Y., Martonchik, J. V., Lattanzio, A., et al. (2004). Intercomparison of surface albedo products from various spaceborne sensors. Proceedings of the Workshop on Inter-Comparison of Large Scale Optical and Infrared Sensors, October 12-14 2004. Noordwijk, The Netherlands: ESA ESTEC.

Pohl, C., \& Van Genderen, J. L. (1998). Multisensor image fusion in remote sensing: Concepts, methods and applications. International Journal of Remote Sensing, 19(5), $823-854$.

Powell, S. L., Pflugmacher, D., Kirschbaum, A. A., Kim, Y., \& Cohen, W. B. (2007). Moderate resolution remote sensing alternatives: A review of Landsat-like sensors and their applications. Journal of Applied Remote Sensing, 1, 012506, doi:10.1117/12.785479.

Roy, D. P. (2000). The impact of misregistration upon composited wide field of view satellite data and implications for change detection. IEEE Transactions on Geoscience and Remote Sensing, 38(4), 2017-2032.

Roy, D. P., Ju, J., Lewis, P., Schaaf, C., Gao, F., Hansen, M., et al. (2008). Multi-temporal MODIS-Landsat data fusion for relative radiometric normalization, gap filling, and prediction of Landsat data. Remote Sensing of Environment, 112(6), 3112-3130.

Roy, D. P., Ju, J., Kline, K., Scaramuzza, P. L., Kovalskyy, V., Hansen, M., et al. (2010). Webenabled Landsat data (WELD): Landsat ETM + composited mosaics of the conterminous United States. Remote Sensing of Environment, 114(1), 35-49.

Schaaf, C., Gao, F., Strahler, A., Lucht, W., Li, X., Tsang, T., et al. (2002). First operational BRDF, albedo and nadir reflectance products from MODIS. Remote Sensing of Environment, 83(1-2), 135-148.

Song, C., Woodcock, C. E., Seto, K. C., Lenney, M. P., \& Macomber, S. A. (2001). Classification of change detection using Landsat TM data: When and how to correct atmospheric effects? Remote Sensing of Environment, 75(2), 230-244.

Storey, J., Scaramuzza, P., Schmidt, G., \& Barsi, J. (2005). Landsat-7 scan line corrector-off gap filled product development. In Proceedings of Pecora 16 Global Priorities in Land Remote Sensing, October 23-27 2005, Sioux Falls, South Dakota. American Society for Photogrammetry and Remote Sensing. http://www.asprs.org/ publications/proceedings/pecora16/Storey_J.pdf.

Toivonen, T., Kalliola, R., Ruokolainen, K., \& Malik, R. N. (2006). Across-path DN gradient in Landsat TM imagery of Amazonian forests: A challenge for image interpretation and mosaicing. Remote Sensing of Environment, Vol. 100(No. 4), 550-562.

Townshend, J. R. G., \& Justice, C. O. (1988). Selecting the spatial-resolution of satellite sensors required for global monitoring of land transformations. International Journal of Remote Sensing, 9(2), 187-236.

Ungar, S. G., Pearlman, J. S., Mendenhall, J. A., \& Reuter, D. (2003). Overview of the Earth Observing One (EO-1) mission. IEEE Transactions on Geoscience and Remote Sensing, 41(6), 1149-1159.

USGS (2008). Imagery for Everyone: Timeline set to release entire USGS Landsat archive at no charge. USGS Technical Announcement, April 21 2008. Available from. http:// landsat.usgs.gov/documents/USGS_Landsat_Imagery_Release.pdf [accessed (August $25,2010)]$.

USGS (2009). Landsat-5 Anomaly-August 13, 2009. Available from. http://landsat.gsfc. nasa.gov/news/news-archive/news_0225.html [accessed (August 25, 2010)].

van Leeuwen, W. J. D., Orr, B. J., Marsh, S. E., \& Herrmann, S. M. (2006). Multi-sensor NDVI data continuity: Uncertainties and implications for vegetation monitoring applications. Remote Sensing of Environment, 100(1), 67-81.

Woodcock, C. E., Allen, R., Anderson, M., Belward, A., Bindschadler, R., Cohen, W., et al. (2008). Free access to Landsat imagery. Science, Vol. 320(No. 5874), 1011.

Wulder, M. A., Ortlepp, S. M., White, J. C., \& Maxwell, S. (2008a). Evaluation of Landsat-7 SLC-off image products for forest change detection. Canadian Journal of Remote Sensing, 34(2), 93-99.

Wulder, M. A., White, J. C., Goward, S. N., Masek, J. G., Irons, J. R., Herold, M., et al. (2008b). Landsat continuity: Issues and opportunities for land cover monitoring. Remote Sensing of Environment, Vol. 112(No. 3), 955-969.

Wulder, M. A., Butson, C. R., \& White, J. C. (2008c). Cross-sensor change detection over a forested landscape: Options to enable continuity of medium spatial resolution measures. Remote Sensing of Environment, 112(3), 796-809.

Wulder, M. A., White, J. C., Gillis, M. D., Walsworth, N., Hansen, M. C., \& Potapov, P. (2010). Multi-scale satellite and spatial information and analysis framework in support of a large-area forest monitoring and inventory update. Environmental Monitoring and Assessment: Vol. 170, No. 1-4. pp. 417-433. (doi:10.1007/s10661-009-1243-8; Open Access: http://www.springerlink.com/content/4652210u416431x8/).

Xiong, X., Che, N., \& Barnes, W. (2005). Terra MODIS on-orbit spatial characterization and performance. IEEE Transactions on Geoscience and Remote Sensing, 43(2), $355-365$. 